\title{
Classification of Ordered Type Soliton Metric Lie Algebras by a Computational Approach
}

\author{
Hülya Kadığlu \\ Department of Mathematics Education, Yıldiz Technical University, 34210 Esenler, Istanbul, Turkey \\ Correspondence should be addressed to Hülya Kadığlu; hkadio@yildiz.edu.tr
}

Received 25 February 2013; Accepted 22 April 2013

Academic Editor: Mustafa Bayram

Copyright (C) 2013 Hülya Kadığlu. This is an open access article distributed under the Creative Commons Attribution License, which permits unrestricted use, distribution, and reproduction in any medium, provided the original work is properly cited.

\begin{abstract}
We classify some soliton nilpotent Lie algebras and possible candidates in dimensions 8 and 9 up toisomorphy. We focus on $1<$ $2<\cdots<n$ type of derivations, where $n$ is the dimension of the Lie algebras. We present algorithms to generate possible algebraic structures.
\end{abstract}

\section{Introduction}

In this paper, we compute and classify $n$-dimensional $(n=$ 8 , 9) nilsoliton metric Lie algebras with eigenvalue type $1<2<\cdots<n$, which will be called "ordered type of Lie algebra" throughout this paper. We use MATLAB to achieve this goal. In the literature, six-dimensional nilpotent Lie algebras have been classified by algorithmic approaches [1]. In dimension seven and lower, nilsoliton metric Lie algebras have been classified [2-9]. Summary and details of some other classifications can be found in [10]. In our paper, we focus on dimensions eight and nine. We note that we have found that our algorithm gives consistent results with the literature in lower dimensions. We use a computational procedure that is similar to the one that we have used in our previous paper [4].

In our previous paper, we classified all the soliton and nonsoliton metric Lie algebras where the corresponding Gram matrix is invertible and of dimensions 7 and 8 up to isomorphism. If corresponding Gram matrix is invertible, then the soliton metric condition $U v=$ [1] has a unique solution. So in this case, it is easy to check if the algebra is soliton or not. But in noninvertible case, there is more than one solution. Therefore it is hard to guess if one of the solutions provides the soliton condition without solving Jacobi identity which is nonlinear. On the other hand, it may be easy if we can eliminate some algebras which admit a derivation $D$ that does not have ordered eigenvalues without solving the following soliton metric condition $U v=$ [1]. For this, we prove that if the nilpotent Lie algebra admits a soliton metric with corresponding Gram matrix of $\eta$ being noninvertible, all the solutions of $U v=$ [1] have a unique derivation. This theorem allows us to omit several cases that come from nonordered eigenvalues without considering Jacobi identity.

This paper is organized as follows. In Section 2, we provide some preliminaries that we use for our classifications. In Section 3, we give specific Jacobi identity conditions for Lie algebras up to dimension nine. This allows us to decide whether the Lie algebra has a soliton metric or not. In Section 4, we give details of our classifications with specific examples and provide algorithmic procedures. Section 5 contains our concluding remarks.

\section{Preliminaries}

Let $\left(\eta_{\mu}, Q\right)$ be a metric algebra, where $\mu \in \Lambda^{2} \eta \otimes \eta^{*}$. Let $B=\left\{X_{i}\right\}_{i=1}^{n}$ be a $Q$-orthonormal basis of $\eta_{\mu}$ (we always assume that bases are ordered). The nil-Ricci endomorphism $\mathrm{Ric}_{\mu}$ is defined as $\left\langle\operatorname{Ric}_{\mu} X, Y\right\rangle=\operatorname{ric}_{\mu}(X, Y)$, where

$$
\begin{aligned}
\operatorname{ric}_{\mu}(X, Y)= & -\frac{1}{2} \sum_{i=1}^{n}\left\langle\left[X, X_{i}\right],\left[Y, X_{i}\right]\right\rangle \\
& +\frac{1}{4} \sum_{i=1}^{n}\left\langle\left[X_{i}, X_{j}\right], X\right\rangle\left\langle\left[X_{i}, X_{j}\right], Y\right\rangle
\end{aligned}
$$


for $X, Y \in \eta$ (we often write an inner product $Q(\cdot, \cdot)$ as $\langle\cdot, \cdot\rangle)$. When $\eta$ is a nilpotent Lie algebra, the nil-Ricci endomorphism is the Ricci endomorphism. If all elements of the basis are eigenvectors for the nil-Ricci endomorphism $\mathrm{Ric}_{\mu}$, we call the orthonormal basis a Ricci eigenvector basis.

Now we define some combinatorial objects associated to a set of integer triples $\Lambda \subset\{(i, j, k) \mid 1 \leq i, j, k \leq n\}$. For $1 \leq i, j, k \leq n$, define $1 \times n$ row vector $y_{i j}^{k}$ to be $\epsilon_{i}^{T}+\epsilon_{j}^{T}-\epsilon_{k}^{t}$, where $\{\epsilon\}_{i=1}^{n}$ is the standard orthonormal basis for $\mathbb{R}^{n}$. We call the vectors in $\left\{y_{i j}^{k} \mid(i, j, k) \in \Lambda\right\}$ root vectors for $\Lambda$. Let $y_{1}, y_{2}, \ldots, y_{m}$ (where $m=|\Lambda|$ ) be an enumeration of the root vectors in dictionary order. We define root matrix $Y_{\Lambda}$ for $\Lambda$ to be the $m \times n$ matrix whose rows are the root vectors $y_{1}, y_{2}, \ldots, y_{m}$. The Gram matrix $U_{\Lambda}$ for $\Lambda$ is the $m \times m$ matrix defined by $U_{\Lambda}=Y_{\Lambda} Y_{\Lambda}^{T}$; the $(i, j)$ entry of $U_{\Lambda}$ is the inner product of the $i$ th and $j$ th root vectors. It is easy to see that $U$ is a symmetric matrix. It has the same rank as the root matrix; that is, $\operatorname{Rank}\left(U_{\Lambda}\right)=\operatorname{Rank}\left(Y_{\Lambda}\right)$. Diagonal elements of $U$ are all three, and the off-diagonal entries of $U$ are in the set $\{-2,-1,0,1,2\}$. For more information, see [11]. Let $D$ have distinct real positive eigenvalues, and let $\Lambda$ index the structure constants for $\eta$ with respect to eigenvector basis $B$. If $\left(i_{1}, j_{1}, k_{1}\right) \in \Lambda$ and $\left(i_{2}, j_{2}, k_{2}\right) \in \Lambda$, then $\left\langle y_{i_{1}, j_{1}}^{k_{1}}, y_{i_{2}, j_{2}}^{k_{2}}\right\rangle \neq 2$. Thus $U$ does not contain two as an entry [4].

Lemma 1. Let $(\eta, Q)$ be an n-dimensional inner product space, and let $\mu$ be an element of $\Lambda^{2} \eta^{*} \otimes \eta$. Suppose that $\eta_{\mu}$ admits a symmetric derivation $D$ having $n$ distinct eigenvalues $0<\lambda_{1}<$ $\lambda_{2}<\cdots<\lambda_{n}$ with corresponding orthonormal eigenvectors $X_{1}, X_{2}, \ldots, X_{n}$. Let $\alpha_{i j}^{k}$ denote the structure constants for $\eta$ with respect to the ordered basis $B=\left\{X_{i}\right\}_{i=1}^{n}$. Let $1 \leq i<j \leq n$. Then

(1) if there is some $k \in\{1,2, \ldots, n\}$ such that $\lambda_{k}=\lambda_{i}+\lambda_{j}$, then $\left[X_{i}, X_{j}\right]$ is a scalar multiple of $X_{k}$; otherwise $X_{i}$ and $X_{j}$ commute;

(2) $\alpha_{i j}^{k} \neq 0$ if and only if $X_{k} \in\left[\eta_{\mu}, \eta_{\mu}\right]$.

Theorem 2 (see [11]). Let $\eta$ be a vector space, and let $B=$ $X_{i=1}^{n}$ be a basis for $\eta$. Suppose that a set of nonzero structure constants $\alpha_{i, j}^{k}$ relative to $B$, indexed by $\Lambda$, defines a skew symmetric product on $\eta$. Assume that if $(i, j, k) \in \Lambda$, then $i<j<k$. Then the algebra is a Lie algebra if and only if whenever there exists $m$ so that the inner product of root vectors $\left\langle y_{i j}^{l}, y_{l k}^{m}\right\rangle=-1$ for triples $(i, j, l)$ and $(l, k, m)$ or $(k, l, m)$ in $\Lambda$, the equation

$$
\sum_{s<m} \alpha_{i, j}^{s} \alpha_{s, k}^{m}+\alpha_{j, k}^{s} \alpha_{s, i}^{m}+\alpha_{k, i}^{s} \alpha_{s, j}^{m}=0
$$

holds. Furthermore, a term of form $\alpha_{i, j}^{l} \alpha_{l, k}^{m}$ is nonzero if and only if $\left\langle y_{i, j}^{l}, y_{l, k}^{m}\right\rangle=-1$

Theorem 3 (see [11]). Let $\left(\eta_{\mu}, Q\right)$ be a metric algebra and $B=$ $\left\{X_{i}\right\}_{i=1}^{n}$ a Ricci eigenvector basis for $\eta_{\mu}$. Let $Y$ be the root matrix for $\eta_{\mu}$. Then the eigenvalues of the nil-Ricci endomorphism are given by

$$
\operatorname{Ric}_{\mu}^{B}=-\frac{1}{2} Y^{T} v
$$

where $v=\left[\alpha^{2}\right]$.

Theorem 4 (see $[4,11])$. Let $(\eta, Q)$ be a nonabelian metric algebra with Ricci eigenvector basis $B$. The following are equivalent.

(1) $\left(\eta_{\mu}, Q\right)$ satisfies the nilsoliton condition with nilsoliton constant $\beta$.

(2) The eigenvalue vector $V_{D}$ for $D=$ Ric $-\beta$ Id with respect to $B$ lies in the kernel of the root matrix for $\left(\eta_{\mu}, Q\right)$ with respect to $B$.

(3) For noncommuting eigenvectors $X$ and $Y$ for the nilRicci endomorphism with eigenvalues $\kappa_{X}$ and $\kappa_{Y}$, the bracket $[X, Y]$ is an eigenvector for the nil-Ricci endomorphism with eigenvalue $\kappa_{X}+\kappa_{Y}-\beta$.

(4) $\beta=y_{i j}^{k}$ Ric for all $(i, j, k)$ in $\Lambda\left(\eta_{\mu}, B\right)$.

Theorem 5 (see [4]). Let $\eta$ be an $n$-dimensional nonabelian nilpotent Lie algebra which admits a derivation $D$ having distinct real positive eigenvalues. Let $B$ be a basis consisting of eigenvectors for the derivation $D$, and let $\Lambda$ index the nonzero structure constants with respect to B. Let $U$ be the $m \times m$ Gram matrix. If $U$ is invertible, then the following hold:

(i) $|\Lambda| \leq n-1$;

(ii) if $\left(i_{1}, j_{1}, k_{1}\right) \in \Lambda$ and $\left(i_{2}, j_{2}, k_{2}\right) \in \Lambda$, then $\left\langle y_{i_{1}, j_{1}}^{k_{2}}\right.$, $\left.y_{i_{2}, j_{2}}^{k_{2}}\right\rangle \neq-1$.

\section{Theory}

This section provides some theorems and their proofs that allow us to consider fewer cases for our algoritm. The following theorem gives a pruning method while Gram matrix is noninvertible.

Theorem 6. Let $\eta$ be an $n$-dimensional nilsoliton metric Lie algebra, and $U$ the corresponding Gram matrix which is noninvertible. Then $\operatorname{Ker}\left(Y^{T}\right)=\operatorname{Ker}(U)$. Furthermore all of the solutions of $U v=[1]$ correspond to a unique derivation.

Proof. Since rank of a matrix is equal to the rank of its Gram matrix, then $p=\operatorname{Rank}(Y)=\operatorname{Rank}(U)$. Let $U: \mathbb{R}^{p} \rightarrow \mathbb{R}^{p}$ and $Y^{T}: \mathbb{R}^{p} \rightarrow \mathbb{R}^{n}$ denote the linear functions (with respect to the standard basis) that correspond to the Gram matrix $U$ and the transpose of the root matrix $Y$, respectively. Since $\operatorname{Rank}(U)=\operatorname{Rank}(Y)=\operatorname{Rank}\left(Y^{T}\right)$ and by rank-nullity theorem, we have

$$
\operatorname{Ker}(U)=\operatorname{Ker}\left(Y^{T}\right) \text {. }
$$

Let $v$ be a particular solution and $v_{0}$ the last column of reduced row echelon matrix $[U,[1]]$. Then $v_{0}$ is also a solution 
of $U v_{0}=[1]$. Therefore $U\left(v-v_{0}\right)=0$; that is, $\left(v-v_{0}\right) \in \operatorname{Ker}(U)$. Using (4), then $\left(v-v_{0}\right) \in \operatorname{Ker}\left(Y^{T}\right)$. For the solution $v_{0}$, suppose that we denote $D_{0}$ for the Nikolayevsky derivation, Ric $_{0}$ for the Ricci tensor, and $\beta_{0}$ for the soliton constant. Then using (3), we have

$$
\left(\operatorname{Ric}-\operatorname{Ric}_{0}\right)=\frac{1}{2} Y^{t}\left(v-v_{0}\right)=0 .
$$

Then Ric $=\operatorname{Ric}_{0}$. Using Theorem 4, we have $\beta=\beta_{0}$, which implies that $D=D_{0}$.

Lemma 7. If nilsoliton metric Lie algebra $\eta$ has ordered type of derivations $1<2<\cdots<n$, then its index set $\Lambda$ consists of triples $(i, j, i+j)$.

Proof. If $V_{D}=\left(\lambda_{1}, \lambda_{2}, \ldots, \lambda_{n}\right)^{T}$ is the eigenvalue vector of $D$ with eigenvector basis $B=\left\{X_{i}\right\}_{i=1}^{n}$ for $\eta$, then by Theorem 4 , $V_{D}$ lies in the kernel of $Y$. Thus for each element $(i, j, k) \in \Lambda$, $\lambda_{i}+\lambda_{j}-\lambda_{k}=0$; that is, $\lambda_{i}+\lambda_{j}=\lambda_{k}$. By Lemma $1,\left[X_{i}, X_{j}\right]=$ $\lambda X_{k}$ for some $\lambda \in \mathbb{R}$. Since $\lambda_{i}=i$ for all $i \in\{1,2, \ldots, n\}, k=$ $i+j$ and $\left[X_{i}, X_{j}\right]=\lambda X_{i+j}$. Hence, the index set for ordered type of derivations is of form $(i, j, i+j)$.

The next corollary describes the index triples $(i, j, k)$ and the Jacobi identity for algebras with ordered type of derivations.

Corollary 8. The algebra $\eta$ is a Lie algebra if and only if for all pairs of form $(i, j, l)$ and $(l, k, m)$ or $(i, j, l)$ and $(k, l, m)$ in $\Lambda_{B}$ with $k \notin\{i, j\}$ and for all $m \geq \max \{i+3, j+2,5\}$, the following equation holds:

$$
\sum_{3 \leq s<m, s \notin\{i, j, k\}} \alpha_{i, j}^{s} \alpha_{s, k}^{m}+\alpha_{j, k}^{s} \alpha_{s, i}^{m}+\alpha_{k, i}^{s} \alpha_{s, j}^{m}=0 .
$$

If in addition $\lambda_{i}=i$ for $i=1, \ldots, n$, then the algebra $\eta$ is a Lie algebra if and only if for all pairs of form $(i, j, i+j)$ and $(i+j, k, i+j+k)$ or $(i, j, i+j)$ and $(k, i+j, i+j+k)$ in $\Lambda_{B}$ with $k \notin\{i, j, i+j\}$ and for all $m=i+j+k \geq \max \{2 i+2, j+2,6\}$, the equation

$$
\sum_{4 \leq s<m, s \notin\{i, j\}} \alpha_{i, j}^{s} \alpha_{s, k}^{m}+\alpha_{j, k}^{s} \alpha_{s, i}^{m}+\alpha_{k, i}^{s} \alpha_{s, j}^{m}=0
$$

holds.

Proof. By Theorem 7 of [11], the algebra $\eta_{\mu}$ defined by $\mu$ is a Lie algebra if and only if whenever there exists $m$ so that $\left\langle y_{i j}^{l}, y_{l k}^{m}\right\rangle=-1$ for triples $(i, j, l)$ and $(l, k, m)$ or $(k, l, m)$ in $\Lambda_{B},(2)$

$$
\sum_{s<m} \alpha_{i, j}^{s} \alpha_{s, k}^{m}+\alpha_{j, k}^{s} \alpha_{s, i}^{m}+\alpha_{k, i}^{s} \alpha_{s, j}^{m}=0
$$

holds. Furthermore, if $i, j$, and $k$ are distinct, the product $\alpha_{i j}^{l} \alpha_{l k}^{m}$ is nonzero if and only if $\left\langle y_{i j}^{l}, y_{l k}^{m}\right\rangle=-1$.

Suppose that $\left\langle y_{i j}^{l}, y_{l k}^{m}\right\rangle=-1$ for $(i, j, l) \in \Lambda_{B}$ and $(l, k, m)$ or $(k, l, m)$ in $\Lambda_{B}$. By definition of $\Lambda_{B}$, we have $i<j$. By Lemma $1, j<l, l<m$, and $k<m$. Since $i<j<l<m$, we know that $m \geq i+3$. Similarly, $j<l<m$ implies that $j+2 \leq m$. If $i=k$ or $j=k$, then $\left\langle y_{i j}^{l}, y_{l k}^{m}\right\rangle=0$, and so $i, j$, and $k$ must be distinct. Since $i, j, k$, and $l$ are all distinct and less than $m$, we know that $m \geq 5$. Thus an expression of form

$$
\alpha_{i, j}^{s} \alpha_{s, k}^{m}+\alpha_{j, k}^{s} \alpha_{s, i}^{m}+\alpha_{k, i}^{s} \alpha_{s, j}^{m}
$$

is nonzero only if $m \geq \max \{i+3, j+2,5\}$, and $k \notin\{i, j\}$.

Suppose that $\lambda_{i}=i$, and $(i, j, l)$ and $(l, k, m)$ are in the index set. Then from Lemma $7, l=i+j$ which implies that $m=i+j+k$. We know that $1 \leq i<j<l<m$. Then, since $j \geq i+1$ and $k \geq 1$, we have $2 i+2 \leq i+j+k=m$. Since $2 i+2 \leq m, m=5$ implies that $i=1$. So there is no possible $(i, j, k)$, where all $i, j, k$ are distinct and $i<j<m$ with $i+j+k$. Thus if $m=5$, then $\left\langle y_{i j}^{l}, y_{l k}^{m}\right\rangle \neq-1$. Therefore, if $\lambda_{i}=i$, an expression of form

$$
\alpha_{i, j}^{s} \alpha_{s, k}^{m}+\alpha_{j, k}^{s} \alpha_{s, i}^{m}+\alpha_{k, i}^{s} \alpha_{s, j}^{m}
$$

is nonzero only if $m \geq \max \{2 i+2, j+2,6\}$, and $k \notin\{i, j\}$.

By Lemma $1, X_{1}$ and $X_{2}$ are in $\left[\eta_{\mu}, \eta_{\mu}\right]^{\perp}$, and so $\alpha_{r t}^{s} \neq 0$ which implies that $s \geq 3$ and $r \neq s, t \neq s$. Therefore all expressions in (10) with $s<3$ or $s \in\{i, j, k\}$ are identically zero and may be omitted from the summation for any $m$.

The next corollary describes some equations in the structure constants of a nilpotent metric Lie algebra that are equivalent to the Jacobi identity. Each of the terms $\alpha_{i j}^{s} \alpha_{s k}^{m}$ in the following equations corresponds to each of -1 entry in the Gram matrix $U$. Therefore, the following equations are useful for noninvertible case since there is no -1 entry in the Gram matrix for the invertible case.

Corollary 9. Let $\left(\eta_{\mu},\langle\cdots, \cdots\rangle\right)$ be an $n$-dimensional inner product space where $n \leq 9$, and, $\mu$ be an element of $\Lambda^{2} \eta^{*} \otimes \eta$. Suppose that the algebra $\eta_{\mu}$ defined by $\mu$ admits a symmetric derivation $D$ having $n$ eigenvalues $1<2<\cdots<n$ with corresponding orthonormal eigenvectors $X_{1}, X_{2}, \ldots, X_{n} . \alpha_{i, j}^{k}$ denote the structure constants for $\eta$ with respect to the ordered basis $B=\left\{X_{i}\right\}_{i=1}^{n}$, and let $\lambda_{B}$ index the nonzero structure constants as defined in (2). The algebra $\eta_{\mu}$ is a Lie algebra if and only if

$$
\begin{gathered}
\alpha_{13}^{4} \alpha_{42}^{6}+\alpha_{23}^{5} \alpha_{51}^{6}=0 \\
\alpha_{12}^{3} \alpha_{3,4}^{7}-\alpha_{14}^{5} \alpha_{52}^{7}-\alpha_{24}^{6} \alpha_{61}^{7}=0 \\
\alpha_{12}^{3} \alpha_{35}^{8}+\alpha_{14}^{5} \alpha_{53}^{8}+\alpha_{15}^{6} \alpha_{62}^{8}+\alpha_{25}^{7} \alpha_{71}^{8}+\alpha_{34}^{7} \alpha_{71}^{8}=0 \\
\alpha_{12}^{3} \alpha_{36}^{9}+\alpha_{13}^{4} \alpha_{45}^{9}-\alpha_{23}^{5} \alpha_{45}^{9}-\alpha_{24}^{6} \alpha_{36}^{9}-\alpha_{34}^{7} \alpha_{27}^{9}-\alpha_{35}^{8} \alpha_{18}^{9}
\end{gathered}
$$

holds. 
TABle 1: Possible $(i, j, k)$ for $m=6$.

\begin{tabular}{lllll}
\hline Case & $i$ & $j$ & $k$ & PT \\
\hline (a) & 1 & 2 & 3 & - \\
(b) & 1 & 3 & 2 & $\checkmark$ \\
(c) & 1 & 4 & 1 & - \\
(d) & 2 & 3 & 1 & $\checkmark$ \\
(e) & 2 & 4 & 0 & - \\
\hline
\end{tabular}

Proof. Following Lemma 7, the index set consists of elements of form $(i, j, i+j)$. Therefore the number $s$ equals to $i+j$ in the expression $\alpha_{i, j}^{s} \alpha_{s, k}^{m}$. From the previous corollary,

$$
\alpha_{i, j}^{s} \alpha_{s, k}^{m}+\alpha_{j, k}^{s} \alpha_{s, i}^{m}+\alpha_{k, i}^{s} \alpha_{s, j}^{m}
$$

is nonzero only if $m \geq 6$, and $k \notin\{i, j\}$. Also $k \neq i+j$; otherwise $\alpha_{s, k}^{m}=0$.

If $m=6,2 i+2 \leq m$ implies that $i \leq 2$; that is, possible numbers for " $i$ " are 1 and 2 . Possible and not possible $(i, j, k)$ triples, which are being used in

$$
\sum_{3 \leq s<m, s \notin\{i, j, k\}} \alpha_{i, j}^{s} \alpha_{s, k}^{m}+\alpha_{j, k}^{s} \alpha_{s, i}^{m}+\alpha_{k, i}^{s} \alpha_{s, j}^{m}=0
$$

and where $i+j+k=6$, are illustrated in Table 1 . The notations in the table are as follows $\checkmark:=$ yes; $-=$ no; $\mathrm{PT}=$ possible triple.

In the case (a), $i+j=k$, and then it is not a possible triple. In the case (b), $i<j, i, j, k$ are distinct, and $k \neq i+j$. So it is a possible triple. In the case (c), $i=k$, and so it is not a possible triple. In the case (d), $i<j$, and all $i, j, k$ are distinct as $i+j \neq k$. Thus it is a possible triple. In the case (e), $k$ is not a natural number, and so it is not a possible triple. Therefore only possible $(i, j, k)$ triples are $(1,3,2)$ and $(2,3,1)$. Triples $(1,3,2)$ and $(2,3,1)$ correspond to nonzero products $\alpha_{13}^{4} \alpha_{42}^{6}$ and $\alpha_{23}^{5} \alpha_{5,1}^{6}$, respectively. Using the skew-symmetry, (2) turns into the following equation:

$$
\alpha_{13}^{4} \alpha_{24}^{6}+\alpha_{23}^{5} \alpha_{15}^{6}=0
$$

which gives (11). Using the same procedure for $m=7$, possible $(i, j, k)$ triples are $(1,2,4),(1,4,2)$ and $(2,4,1)$, which correspond to nonzero $\alpha_{12}^{3} \alpha_{34}^{7}, \alpha_{14}^{5} \alpha_{52}^{7}$, and $\alpha_{24}^{6} \alpha_{61}^{7}$ respectively. Therefore (12) is obtained. Equations (13) and (14) can be obtained by the same way.

As an illustration, we show how to use the results of this section in the following example.

Example 10. Let $\eta$ be an 8-dimensional algebra with nonzero structure constants relative to eigenvector basis $B$ indexed by

$$
\begin{array}{r}
\Lambda=\{(1,2,3),(1,3,4),(1,4,5),(1,6,7), \\
(2,3,5),(2,6,8),(3,4,7),(3,5,8)\} .
\end{array}
$$

Computation shows that the structure vector $\left[\alpha^{2}\right]$ is a solution to $U v=[1]_{8 \times 1}$ if and only if it is of form

$$
\begin{aligned}
& {\left[\alpha^{2}\right]=\left(\begin{array}{c}
\left(\alpha_{12}^{3}\right)^{2} \\
\left(\alpha_{13}^{4}\right)^{2} \\
\left(\alpha_{14}^{5}\right)^{2} \\
\left(\alpha_{16}^{7}\right)^{2} \\
\left(\alpha_{23}^{5}\right)^{2} \\
\left(\alpha_{26}^{8}\right)^{2} \\
\left(\alpha_{34}^{7}\right)^{2} \\
\left(\alpha_{35}^{8}\right)^{2}
\end{array}\right)=\left(\begin{array}{c}
-\frac{3}{17} \\
\frac{10}{17} \\
\frac{15}{17} \\
-\frac{8}{17} \\
-\frac{5}{17} \\
\frac{12}{17} \\
\frac{7}{17} \\
0 \\
0
\end{array}\right)} \\
& +\left(\begin{array}{c}
1 \\
0 \\
-1 \\
0 \\
0 \\
-1 \\
0 \\
1 \\
0
\end{array}\right)+Y \cdot\left(\begin{array}{c}
1 \\
-1 \\
-1 \\
1 \\
1 \\
-1 \\
-1 \\
0 \\
1
\end{array}\right)
\end{aligned}
$$

Equation (13) from the previous corollary leads

$$
\begin{aligned}
\left(-\frac{3}{17}+X+Y\right) \cdot Y & =\left(\frac{15}{17}-X-Y\right) \cdot Y \\
& \Longrightarrow X+Y=\frac{9}{17} .
\end{aligned}
$$

Moreover, using (12), we find that $X=3 / 17$ and $Y=6 / 17$, which means that $\left(\alpha_{16}^{7}\right)^{2}=-2 / 17$. Thus $\eta$ is not a Lie algebra.

\section{Algorithm and Classifications}

In this section, we describe our computational procedure and give the results in dimensions 8 and 9 .

4.1. Algorithm. Now we describe the algorithm. The following algorithm can be used for both invertible and noninvertible cases.

Input. The input is the integer $n$ which represents the dimension.

Output. The output is two $0-1$ matrices Wsoliton and Uninv listing characteristic vectors for index sets $\Lambda$ of $\Theta_{n}$. The matrix Wsoliton has as its rows all possible characteristic vectors for canonical index sets $\Lambda$ for nilpotent Lie algebras of dimension $n$ with ordered type nonsingular nilsoliton 
TABLE 2: 8-dimensional nilsoliton metric Lie algebras.

\begin{tabular}{|c|c|c|c|}
\hline & Lie bracket & Index & Nullity \\
\hline 1 & $\begin{array}{c}(0,0, \sqrt{274 / 2223} \cdot 12, \sqrt{99 / 764} \cdot 13, \sqrt{527 / 8179} \cdot 14+\sqrt{1532 / 9311} \cdot 23, \sqrt{101 / 2154} \cdot 15+ \\
\sqrt{250 / 4199} \cdot 24, \sqrt{150 / 3151} \cdot 16+\sqrt{110 / 367} \cdot 25+\sqrt{110 / 367} \cdot 34, \sqrt{7 / 17} \cdot 17)\end{array}$ & 6 & 3 \\
\hline 2 & $\begin{array}{c}(0,0, \sqrt{82 / 7253} \cdot 12, \sqrt{4 / 34} \cdot 13, \sqrt{97 / 299} \cdot 14+\sqrt{6 / 34} \cdot 23, \sqrt{1 / 34} \cdot 15+\sqrt{9 / 34} \cdot 24, \sqrt{10 / 34} \cdot 16+ \\
\sqrt{1998 / 12097} \cdot 25+\sqrt{397 / 13917} \cdot 34, \sqrt{728 / 2883} \cdot 17+\sqrt{637 / 4000} \cdot 35)\end{array}$ & 6 & 4 \\
\hline 3 & $\begin{array}{c}(0,0, \sqrt{163 / 702} \cdot 12, \sqrt{263 / 2572} \cdot 13, \sqrt{343 / 1334} \cdot 14+\sqrt{547 / 2871} \cdot 23, \sqrt{175 / 2358} \cdot 15+ \\
\sqrt{160 / 1149} \cdot 24, \sqrt{388 / 2509} \cdot 16+\sqrt{518 / 6185} \cdot 25, \sqrt{43 / 13870} \cdot 17+\sqrt{915 / 2239} \cdot 35)\end{array}$ & 6 & 3 \\
\hline
\end{tabular}

TABLE 3: The 9-dimensional nilsoliton metric Lie algebras of nullities 1 and 2.

\begin{tabular}{|c|c|c|c|}
\hline & Lie bracket & Index & Nullity \\
\hline 1 & $(0,0,0,0, \sqrt{45} \cdot 23, \sqrt{14} \cdot 24, \sqrt{91} \cdot 25+\sqrt{91} \cdot 34, \sqrt{136} \cdot 17+\sqrt{29} \cdot 26+\sqrt{14} \cdot 35, \sqrt{104} \cdot 18+4.27)$ & 4 & 1 \\
\hline 2 & $(0,0,1.12,0,1.14+\sqrt{3} \cdot 23,0, \sqrt{6} \cdot 25+\sqrt{6} \cdot 34, \sqrt{8} \cdot 17+1.26+\sqrt{2} \cdot 35, \sqrt{6} \cdot 18+\sqrt{2} \cdot 27)$ & 5 & 2 \\
\hline$\underline{3}$ & $(0,0, \sqrt{21} \cdot 12,0, \sqrt{21} \cdot 14+\sqrt{39} \cdot 23,0, \sqrt{13} \cdot 16+\sqrt{70} \cdot 25-\sqrt{70} \cdot 34, \sqrt{88} \cdot 17+\sqrt{42} \cdot 35, \sqrt{65} \cdot 18+\sqrt{39} \cdot 27)$ & 5 & 2 \\
\hline
\end{tabular}

derivation whose canonical Gram matrix $U$ is invertible. The matrix Uninv has as its rows all possible characteristic vectors for canonical index sets $\Lambda$ for nilpotent Lie algebras of dimension $n$ with ordered type nonsingular nilsoliton derivation whose canonical Gram matrix $U$ is noninvertible. In the dimensions 8 and 9, there is no example for invertible case. Thus Wsoliton is an empty matrix. Therefore we give the algorithm for the noninvertible case.

Algorithm for the Noninvertible Case. Consider the following.

(i) Enter the dimension $n$.

(ii) Compute the matrix $Z_{n}$.

(iii) Compute the matrix $W$.

(iv) Delete all rows of $W$ containing abelian factor which is the row that represents direct sums of Lie algebras.

(v) Remove all rows of $W$ such that the canonical Gram matrix $U$ associated to the index set $\Lambda$ is invertible.

(vi) Define eigenvalue vector $v_{D}=(1,2,3, \ldots, n)^{T}$ in dimension $n$.

(vii) Remove all rows of $W$ if $v(i)=v_{0}(i) \leq 0$ where $v$ is the general solution of $U_{\Lambda} v=[1]_{m \times 1}$ and $v_{0}$ is the vector that we have defined in the proof of Theorem 6.

(viii) Remove all the rows of $W$ such that the corresponding algebra does not have a derivation of eigenvalue type $1<2<\cdots<n$.

(ix) Remove all the rows of $W$ such that the corresponding algebra does not satisfy Jacobi identity condition, which is obtained in Corollary 9.

After this process, we solve nonlinear systems which follow from Jacobi identity. In order to see how the algorithm works, we give the following example for $n=6$.

Example 11. Let $n=6$. Then

$\Theta_{6}=\{(1,2,3),(1,3,4),(1,4,5),(1,5,6),(2,3,5),(2,4,6)\}$.
TABLE 4: The 8-dimensional nilsoliton metric Lie algebra candidates.

\begin{tabular}{cccc}
\hline & Lie bracket & Index & Nullity \\
\hline 1 & $(0,0,1.12,1.13,1.14+1.23,1.15+1.24$, & 6 & 4 \\
& $1.16+1.34,1.17+1.26+1.35)$ & 6 & \\
2 & $(0,0,1.12,1.13,1.14+1.23,1.15+1.24$, & 6 & 4 \\
& $1.16+1.25,1.17+1.26+1.35)$ & & \\
3 & $(0,0,1.12,1.13,1.14+1.23,1.15+1.24$, & 6 & 4 \\
& $1.16+1.25+1.34,1.17+1.26)$ & & \\
4 & $(0,0,1.12,1.13,1.14+1.23,1.15+1.24$, & 6 & 5 \\
& $1.16+1.25+1.34,1.17+1.26+1.35)$ & & \\
\hline
\end{tabular}

TABLE 5: Number of 9-dimensional nilsoliton metric Lie algebra candidates.

\begin{tabular}{lc}
\hline Nullity & Number of Lie algebras \\
\hline 3 & 98 \\
4 & 81 \\
5 & 45 \\
6 & 22 \\
7 & 7 \\
8 & 1 \\
\hline
\end{tabular}

So, matrix $Z_{6}$ is $6 \times 3$ of form

$$
Z_{6}=\left(\begin{array}{lll}
1 & 2 & 3 \\
1 & 3 & 4 \\
1 & 4 & 5 \\
1 & 5 & 6 \\
2 & 3 & 5 \\
2 & 4 & 6
\end{array}\right)
$$

Since $\left|\Theta_{6}\right|=6$, the matrix $W$ is of size $2^{6} \times 6$ as follows

$$
W_{\Theta}=\left(\begin{array}{cccccc}
0 & 0 & 0 & 0 & 0 & 0 \\
0 & 0 & 0 & 0 & 0 & 1 \\
0 & 0 & 0 & 0 & 1 & 0 \\
\vdots & \vdots & \vdots & \vdots & \vdots & \vdots \\
1 & 1 & 1 & 1 & 1 & 1
\end{array}\right) .
$$


TABLE 6: The 9-dimensional candidates of nullities 6 and 8 .

\begin{tabular}{|c|c|c|c|}
\hline & Lie bracket & Index & Nullity \\
\hline 1 & $(0,0,1.12,1.13,1.14+1.23,0,1.16+1.25+1.34,1.17+1.26+1.35,1.18+1.27+1.36+1.45)$ & 6 & 6 \\
\hline 2 & $(0,0,1.12,1.13,1.14,1.15,1.16+1.25+1.34,1.17+1.26+1.35,1.18+1.27+1.36+1.45)$ & 7 & 6 \\
\hline 3 & $(0,0,1.12,1.13,1.14+1.23,1.15+1.24,1.16+1.34,1.17+1.35,1.18+1.27+1.36+1.45)$ & 7 & 6 \\
\hline 4 & $(0,0,1.12,1.13,1.14+1.23,1.15+1.24,1.16+1.34,1.17+1.26+1.35,1.18+1.36+1.45)$ & 7 & 6 \\
\hline 5 & $(0,0,1.12,1.13,1.14+1.23,1.15+1.24,1.16+1.34,1.17+1.26,1.18+1.27+1.36+1.45)$ & 7 & 6 \\
\hline 6 & $(0,0,1.12,1.13,1.14+1.23,1.15+1.24,1.16+1.34,1.17+1.26+1.35,1.18+1.27+1.45)$ & 7 & 6 \\
\hline 7 & $(0,0,1.12,1.13,1.14+1.23,1.15+1.24,1.16+1.34,1.17+1.26+1.35,1.18+1.27+1.36)$ & 7 & 6 \\
\hline 8 & $(0,0,1.12,1.13,1.14+1.23,1.15+1.24,1.16+1.25+1.34,1.17+1.35,1.18+1.36+1.45)$ & 7 & 6 \\
\hline 9 & $(0,0,1.12,1.13,1.14+1.23,1.15+1.24,1.16+1.25,1.17+1.35,1.18+1.27+1.36+1.45)$ & 7 & 6 \\
\hline 10 & $(0,0,1.12,1.13,1.14+1.23,1.15+1.24,1.16+1.25+1.34,1.17,1.18+1.27+1.36+1.45)$ & 7 & 6 \\
\hline 11 & $(0,0,1.12,1.13,1.14+1.23,1.15+1.24,1.16+1.25+1.34,1.17+1.35,1.18+1.27+1.45)$ & 7 & 6 \\
\hline 12 & $(0,0,1.12,1.13,1.14+1.23,1.15+1.24,1.16+1.25+1.34,1.17+1.35,1.18+1.27+1.36)$ & 7 & 6 \\
\hline 13 & $(0,0,1.12,1.13,1.14+1.23,1.15+1.24,1.16+1.25,1.17+1.26+1.35,1.18+1.36+1.45)$ & 7 & 6 \\
\hline 14 & $(0,0,1.12,1.13,1.14+1.23,1.15+1.24,1.16+1.25+1.34,1.17+1.26,1.18+1.36+1.45)$ & 7 & 6 \\
\hline 15 & $(0,0,1.12,1.13,1.14+1.23,1.15+1.24,1.16+1.25+1.34,1.17+1.26+1.35,1.18+1.45)$ & 7 & 6 \\
\hline 16 & $(0,0,1.12,1.13,1.14+1.23,1.15+1.24,1.16+1.25+1.34,1.17+1.26+1.35,1.18+1.36)$ & 7 & 6 \\
\hline 17 & $(0,0,1.12,1.13,1.14+1.23,1.15+1.24,1.16+1.25,1.17+1.26,1.18+1.27+1.36+1.45)$ & 7 & 6 \\
\hline 18 & $(0,0,1.12,1.13,1.14+1.23,1.15+1.24,1.16+1.25,1.17+1.26+1.35,1.18+1.27+1.45)$ & 7 & 6 \\
\hline 19 & $(0,0,1.12,1.13,1.14+1.23,1.15+1.24,1.16+1.25,1.17+1.26+1.35,1.18+1.27+1.36)$ & 7 & 6 \\
\hline 20 & $(0,0,1.12,1.13,1.14+1.23,1.15+1.24,1.16+1.25+1.34,1.17+1.26,1.18+1.27+1.45)$ & 7 & 6 \\
\hline 21 & $(0,0,1.12,1.13,1.14+1.23,1.15+1.24,1.16+1.25+1.34,1.17+1.26,1.18+1.27+1.36)$ & 7 & 6 \\
\hline 22 & $(0,0,1.12,1.13,1.14+1.23,1.15+1.24,1.16+1.25+1.34,1.17+1.26+1.35,1.18+1.27)$ & 7 & 6 \\
\hline 23 & $(0,0,1.12,1.13,1.14+1.23,1.15+1.24,1.16+1.25+1.34,1.17+1.26+1.35,1.18+1.27+1.36+1.45)$ & 7 & 8 \\
\hline
\end{tabular}

The first row of $W_{\theta}$ represents empty matrix, row two represents the subset $\{(2,4,6)\}$ of $\Theta_{6}$, and so forth. Eliminating rows that represent direct sums, we have 33 rows in $W$ matrix. Therefore none of the rows of $W$ corresponds to Lie algebras that can be written as direct sums. These algebras correspond both to invertible and noninvertible Gram matrices. There is no example for the invertible case. For the noninvertible case, there is one ordered type nilsoliton metric Lie algebra $\eta$. Let $B$ be the eigenvector basis for $\eta$, whose nonzero structure constants are indexed by

$\Lambda_{\eta}=\{(1,2,3),(1,3,4),(1,4,5),(1,5,6),(2,3,5),(2,4,6)\}$

Computation shows that the structure vector $\left[\alpha^{2}\right]$ is a solution to $U v=[1]_{6 \times 1}$ if and only if it is of form

$$
\left[\alpha^{2}\right]=\left(\begin{array}{l}
\left(\alpha_{12}^{3}\right)^{2} \\
\left(\alpha_{13}^{4}\right)^{2} \\
\left(\alpha_{14}^{5}\right)^{2} \\
\left(\alpha_{15}^{6}\right)^{2} \\
\left(\alpha_{23}^{5}\right)^{2} \\
\left(\alpha_{24}^{6}\right)^{2}
\end{array}\right)=\frac{1}{143}\left(\begin{array}{l}
2 \\
1 \\
2 \\
5 \\
5 \\
0
\end{array}\right)+\frac{t}{143}\left(\begin{array}{c}
0 \\
1 \\
0 \\
-1 \\
-1 \\
1
\end{array}\right)
$$

By Corollary 9, $\eta$ satisfies (11). Solving the equation for $t$, we find that

$$
\begin{gathered}
\left(\alpha_{13}^{4} \alpha_{24}^{6}\right)^{2}=\left(\alpha_{23}^{5} \alpha_{15}^{6}\right)^{2}, \\
(11+t) t=(55-t)^{2}, \\
t=25 .
\end{gathered}
$$

After rescaling and solving for structure constants from $\left[\alpha^{2}\right]$, we see that letting

$$
\begin{array}{ll}
{\left[X_{1}, X_{2}\right]=\sqrt{22} X_{3},} & {\left[X_{1}, X_{3}\right]=6 X_{4},} \\
{\left[X_{1}, X_{4}\right]=\sqrt{22} X_{5},} & {\left[X_{1}, X_{5}\right]=\sqrt{30} X_{6},} \\
{\left[X_{2}, X_{3}\right]=\sqrt{30} X_{5},} & {\left[X_{2}, X_{4}\right]=5 X_{6}}
\end{array}
$$

defines a nilsoliton metric Lie algebra, previously found in [3].

4.2. Classifications. Classification results for dimensions 8 and 9 appear in Tables 2 and 3, respectively. We use vector notations to represent Lie algebra structures. For example, the list

$(0,0,0,0, \sqrt{45} \cdot 23, \sqrt{14} \cdot 24, \sqrt{91} \cdot 25+\sqrt{91} \cdot 34, \sqrt{136} \cdot 17$

$$
+\sqrt{29} \cdot 26+\sqrt{14} \cdot 35, \sqrt{104} \cdot 18+4 \cdot 27)
$$


TABLE 7: The 9-dimensional nilsoliton metric Lie algebra candidates of nullity 3.

\begin{tabular}{|c|c|c|c|}
\hline & Lie bracket & Index & Nullity \\
\hline 1 & $(0,0,0,0,1.23,1.24,1.25+1.34,1.17+1.26+1.35,1.18,1.27+1.36+1.45)$ & 4 & 3 \\
\hline 2 & $(0,0,1.12,0,1.23,1.24,1.16+1.25+1.34,1.17,1.18+1.27+1.36+1.45)$ & 5 & 3 \\
\hline 3 & $(0,0,1.12,0,1.23,1.24,1.16+1.25+1.34,1.17+1.35,1.18+1.27+1.45)$ & 5 & 3 \\
\hline 4 & $(0,0,1.12,0,1.23,1.24,1.16+1.25+1.34,1.17+1.35,1.18+1.27+1.36)$ & 5 & 3 \\
\hline 5 & $(0,0,1.12,0,1.23,1.24,1.16+1.25+1.34,1.17+1.26,1.18+1.36+1.45)$ & 5 & 3 \\
\hline 6 & $(0,0,1.12,0,1.23,1.24,1.16+1.25+1.34,1.17+1.26+1.35,1.18+1.45)$ & 5 & 3 \\
\hline 7 & $(0,0,1.12,0,1.23,1.24,1.16+1.25+1.34,1.17+1.26+1.35,1.18+1.36)$ & 5 & 3 \\
\hline 8 & $(0,0,1.12,0,1.23,1.24,1.16+1.25+1.34,1.17+1.26,1.18+1.27+1.45)$ & 5 & 3 \\
\hline 9 & $(0,0,1.12,0,1.23,1.24,1.16+1.25+1.34,1.17+1.26,1.18+1.27+1.36)$ & 5 & 3 \\
\hline 10 & $(0,0,1.12,0,1.23,1.24,1.16+1.25+1.34,1.17+1.26+1.35,1.18+1.27)$ & 5 & 3 \\
\hline 11 & $(0,0,1.12,0,1.14+1.23,0,1.25+1.34,1.17+1.26,1.18+1.27+1.36+1.45)$ & 5 & 3 \\
\hline 12 & $(0,0,1.12,0,1.14+1.23,0,1.25+1.34,1.17+1.26+1.35,1.18+1.27+1.45)$ & 5 & 3 \\
\hline 13 & $(0,0,1.12,0,1.14+1.23,0,1.25+1.34,1.17+1.26+1.35,1.18+1.27+1.36)$ & 5 & 3 \\
\hline 14 & $(0,0,1.12,0,1.14+1.23,1.24,1.25+1.34,1.17,1.18+1.27+1.36+1.45)$ & 5 & 3 \\
\hline 15 & $(0,0,1.12,0,1.14+1.23,1.24,1.25+1.34,1.17+1.35,1.18+1.27+1.36)$ & 5 & 3 \\
\hline 16 & $(0,0,1.12,0,1.14+1.23,1.24,1.25+1.34,1.17+1.26,1.18+1.36+1.45)$ & 5 & 3 \\
\hline 17 & $(0,0,1.12,0,1.14+1.23,1.24,1.25+1.34,1.17+1.26+1.35,1.18+1.45)$ & 5 & 3 \\
\hline 18 & $(0,0,1.12,0,1.14+1.23,1.24,1.25+1.34,1.17+1.26+1.35,1.18+1.45)$ & 5 & 3 \\
\hline 19 & $(0,0,1.12,0,1.14+1.23,1.24,1.25+1.34,1.17+1.26,1.18+1.27+1.45)$ & 5 & 3 \\
\hline 20 & $(0,0,1.12,0,1.14+1.23,1.24,1.25+1.34,1.17+1.26,1.18+1.27+1.36)$ & 5 & 3 \\
\hline 21 & $(0,0,1.12,0,1.14+1.23,1.24,1.25+1.34,1.17+1.26+1.35,1.18+1.27)$ & 5 & 3 \\
\hline 22 & $(0,0,1.12,0,1.14+1.23,0,1.16+1.25+1.34,1.17,1.18+1.27+1.36+1.45)$ & 5 & 3 \\
\hline 23 & $(0,0,1.12,0,1.14+1.23,0,1.16+1.25+1.34,1.17+1.35,1.18+1.27+1.45)$ & 5 & 3 \\
\hline 24 & $(0,0,1.12,0,1.14+1.23,0,1.16+1.25+1.34,1.17+1.35,1.18+1.27+1.36)$ & 5 & 3 \\
\hline 25 & $(0,0,1.12,0,1.14+1.23,0,1.16+1.25+1.34,1.17+1.26,1.18+1.36+1.45)$ & 5 & 3 \\
\hline 26 & $(0,0,1.12,0,1.14+1.23,0,1.16+1.25+1.34,1.17+1.26+1.35,1.18+1.45)$ & 5 & 3 \\
\hline 27 & $(0,0,1.12,0,1.14+1.23,0,1.16+1.25+1.34,1.17+1.26,1.18+1.27+1.45)$ & 5 & 3 \\
\hline 28 & $(0,0,1.12,0,1.14+1.23,0,1.16+1.25+1.34,1.17+1.26,1.18+1.27+1.36)$ & 5 & 3 \\
\hline 29 & $(0,0,1.12,0,1.14+1.23,0,1.16+1.25+1.34,1.17+1.26+1.35,1.18+1.27)$ & 5 & 3 \\
\hline 30 & $(0,0,1.12,0,1.14+1.23,1.24,1.16+1.34,1.17+1.35,1.18+1.27+1.45)$ & 4 & 3 \\
\hline 31 & $(0,0,1.12,0,1.14+1.23,1.24,1.16+1.34,1.17+1.35,1.18+1.27+1.36)$ & 4 & 3 \\
\hline 32 & $(0,0,1.12,0,1.14+1.23,1.24,1.16+1.34,1.17+1.26+1.35,1.18+1.45)$ & 4 & 3 \\
\hline 33 & $(0,0,1.12,0,1.14+1.23,1.24,1.16+1.34,1.17+1.26+1.35,1.18+1.36)$ & 4 & 3 \\
\hline 34 & $(0,0,1.12,0,1.14+1.23,1.24,1.16+1.34,1.17+1.26+1.35,1.18+1.27)$ & 4 & 3 \\
\hline 35 & $(0,0,1.12,1.13,1.23,0,1.16+1.25,1.17+1.26+1.35,1.27+1.36+1.45)$ & 4 & 3 \\
\hline 36 & $(0,0,1.12,1.13,1.14+1.23,0,1.25+1.34,1.26+1.35,1.18+1.27+1.45)$ & 5 & 3 \\
\hline 37 & $(0,0,1.12,1.13,1.14+1.23,0,1.25+1.34,1.26+1.35,1.18+1.27+1.36)$ & 5 & 3 \\
\hline 38 & $(0,0,1.12,1.13,1.14+1.23,0,1.25+1.34,1.17+1.26,1.27+1.36+1.45)$ & 5 & 3 \\
\hline 39 & $(0,0,1.12,1.13,1.14+1.23,0,1.25+1.34,1.17+1.26+1.35,1.27+1.45)$ & 5 & 3 \\
\hline 40 & $(0,0,1.12,1.13,1.14+1.23,0,1.25+1.34,1.17+1.26+1.35,1.27+1.36)$ & 5 & 3 \\
\hline 41 & $(0,0,1.12,1.13,1.14,0,1.25+1.34,1.17+1.26,1.18+1.27+1.36+1.45)$ & 5 & 3 \\
\hline 42 & $(0,0,1.12,1.13,1.14,0,1.25+1.34,1.17+1.26+1.35,1.18+1.27+1.45)$ & 6 & 3 \\
\hline 43 & $(0,0,1.12,1.13,1.14,0,1.25+1.34,1.17+1.26+1.35,1.18+1.27+1.36)$ & 6 & 3 \\
\hline 44 & $(0,0,1.12,1.13,1.14+1.23,0,1.25+1.34,1.17+1.26,1.18+1.36+1.45)$ & 6 & 3 \\
\hline 45 & $(0,0,1.12,1.13,1.14+1.23,0,1.25+1.34,1.17+1.26+1.35,1.18+1.45)$ & 6 & 3 \\
\hline 46 & $(0,0,1.12,1.13,1.14+1.23,0,1.25+1.34,1.17+1.26,1.18+1.27+1.45)$ & 6 & 3 \\
\hline 47 & $(0,0,1.12,1.13,1.14+1.23,0,1.25+1.34,1.17+1.26,1.18+1.27+1.36)$ & 6 & 3 \\
\hline 48 & $(0,0,1.12,1.13,1.14+1.23,0,1.25+1.34,1.17+1.26+1.35,1.18+1.27)$ & 6 & 3 \\
\hline 49 & $(0,0,1.12,1.13,1.14+1.23,0,1.16+1.25+1.34,1.26+1.35,1.27+1.45)$ & 5 & 3 \\
\hline 50 & $(0,0,1.12,1.13,1.14+1.23,0,1.16+1.25+1.34,1.26+1.35,1.27+1.36)$ & 5 & 3 \\
\hline 51 & $(0,0,1.12,1.13,1.14+1.23,0,1.16+1.25+1.34,1.35,1.18+1.27+1.45)$ & 5 & 3 \\
\hline 52 & $(0,0,1.12,1.13,1.14+1.23,0,1.16+1.25+1.34,1.35,1.18+1.27+1.36)$ & 5 & 3 \\
\hline
\end{tabular}


TABLe 7: Continued.

\begin{tabular}{|c|c|c|c|}
\hline & Lie bracket & Index & Nullity \\
\hline 53 & $(0,0,1.12,1.13,1.14+1.23,0,1.16+1.25+1.34,1.26+1.35,1.18+1.45)$ & 5 & 3 \\
\hline 54 & $(0,0,1.12,1.13,1.14+1.23,0,1.16+1.25+1.34,1.26+1.35,1.18+1.36)$ & 5 & 3 \\
\hline 55 & $(0,0,1.12,1.13,1.14+1.23,0,1.16+1.25+1.34,1.26+1.35,1.18+1.27)$ & 5 & 3 \\
\hline 56 & $(0,0,1.12,1.13,1.14,0,1.16+1.25+1.34,1.17+1.26,1.27+1.36+1.45)$ & 5 & 3 \\
\hline 57 & $(0,0,1.12,1.13,1.14,0,1.16+1.25+1.34,1.17+1.26+1.35,1.27+1.45)$ & 5 & 3 \\
\hline 58 & $(0,0,1.12,1.13,1.14+1.23,0,1.16+1.25+1.34,1.17,1.27+1.36+1.45)$ & 5 & 3 \\
\hline 59 & $(0,0,1.12,1.13,1.14+1.23,0,1.16+1.25+1.34,1.17+1.35,1.27+1.45)$ & 5 & 3 \\
\hline 60 & $(0,0,1.12,1.13,1.14,0,1.16+1.25+1.34,1.17,1.18+1.27+1.36+1.45)$ & 6 & 3 \\
\hline 61 & $(0,0,1.12,1.13,1.14,0,1.16+1.25+1.34,1.17+1.35,1.18+1.27+1.45)$ & 6 & 3 \\
\hline 62 & $(0,0,1.12,1.13,1.14,0,1.16+1.25+1.34,1.17+1.35,1.18+1.27+1.36)$ & 6 & 3 \\
\hline 63 & $(0,0,1.12,1.13,1.14,0,1.16+1.25+1.34,1.17+1.26,1.18+1.27+1.45)$ & 6 & 3 \\
\hline 64 & $(0,0,1.12,1.13,1.14,0,1.16+1.25+1.34,1.17+1.26,1.18+1.27+1.36)$ & 6 & 3 \\
\hline 65 & $(0,0,1.12,1.13,1.14,0,1.16+1.25+1.34,1.17+1.26+1.35,1.18+1.27)$ & 6 & 3 \\
\hline 66 & $(0,0,1.12,1.13,1.14+1.23,0,1.16+1.25+1.34,1.17,1.18+1.36+1.45)$ & 6 & 3 \\
\hline 67 & $(0,0,1.12,1.13,1.14+1.23,0,1.16+1.25+1.34,1.17+1.35,1.18+1.45)$ & 6 & 3 \\
\hline 68 & $(0,0,1.12,1.13,1.14+1.23,0,1.16+1.25+1.34,1.17+1.35,1.18+1.45)$ & 6 & 3 \\
\hline
\end{tabular}

TABLE 8: The 9-dimensional nilsoliton metric Lie algebra candidates of nullity 3.

\begin{tabular}{|c|c|c|c|}
\hline & Lie bracket & Index & Nullity \\
\hline 69 & $(0,0,1.12,1.13,1.14+1.23,0,1.16+1.25+1.34,1.17,1.18+1.27+1.45)$ & 6 & 3 \\
\hline 70 & $(0,0,1.12,1.13,1.14+1.23,0,1.16+1.25+1.34,1.17,1.18+1.27+1.36)$ & 6 & 3 \\
\hline 71 & $(0,0,1.12,1.13,1.14+1.23,0,1.16+1.25+1.34,1.17+1.35,1.18+1.27)$ & 6 & 3 \\
\hline 72 & $(0,0,1.12,1.13,1.14+1.23,0,1.16+1.25+1.34,1.17+1.26,1.18+1.45)$ & 6 & 3 \\
\hline 73 & $(0,0,1.12,1.13,1.14+1.23,0,1.16+1.25+1.34,1.17+1.26+1.35,1.18)$ & 6 & 3 \\
\hline 74 & $(0,0,1.12,1.13,1.14,1.15,1.25+1.34,1.26,1.18+1.27+1.36+1.45)$ & 6 & 3 \\
\hline 75 & $(0,0,1.12,1.13,1.14,1.15,1.25+1.34,1.26+1.35,1.18+1.27+1.45)$ & 6 & 3 \\
\hline 76 & $(0,0,1.12,1.13,1.14,1.15,1.25+1.34,1.26+1.35,1.18+1.27+1.36)$ & 6 & 3 \\
\hline 77 & $(0,0,1.12,1.13,1.14,1.15,1.25+1.34,1.17,1.18+1.27+1.36+1.45)$ & 6 & 3 \\
\hline 78 & $(0,0,1.12,1.13,1.14,1.15,1.25+1.34,1.17+1.35,1.18+1.27+1.36)$ & 6 & 3 \\
\hline 79 & $(0,0,1.12,1.13,1.14,1.15,1.16+1.25+1.34,1.26,1.27+1.36+1.45)$ & 7 & 3 \\
\hline 80 & $(0,0,1.12,1.13,1.14,1.15,1.16+1.25+1.34,1.26+1.35,1.27+1.45)$ & 7 & 3 \\
\hline 81 & $(0,0,1.12,1.13,1.14,1.15,1.16+1.25+1.34,1.26+1.35,1.27+1.36)$ & 7 & 3 \\
\hline 82 & $(0,0,1.12,1.13,1.14,1.15,1.16+1.25+1.34,1.35,1.18+1.27+1.45)$ & 7 & 3 \\
\hline 83 & $(0,0,1.12,1.13,1.14,1.15,1.16+1.25+1.34,1.35,1.18+1.27+1.36)$ & 7 & 3 \\
\hline 84 & $(0,0,1.12,1.13,1.14,1.15,1.16+1.25+1.34,1.26,1.18+1.27+1.45)$ & 7 & 3 \\
\hline 85 & $(0,0,1.12,1.13,1.14,1.15,1.16+1.25+1.34,1.26,1.18+1.27+1.36)$ & 7 & 3 \\
\hline 86 & $(0,0,1.12,1.13,1.14,1.15,1.16+1.25+1.34,1.26+1.35,1.18+1.27)$ & 7 & 3 \\
\hline 87 & $(0,0,1.12,1.13,1.14,1.15,1.16+1.25+1.34,1.17,1.27+1.36+1.45)$ & 6 & 3 \\
\hline 88 & $(0,0,1.12,1.13,1.14,1.15,1.16+1.25+1.34,1.17+1.35,1.27+1.45)$ & 6 & 3 \\
\hline 89 & $(0,0,1.12,1.13,1.14,1.15,1.16+1.25+1.34,1.17+1.35,1.27+1.36)$ & 6 & 3 \\
\hline 90 & $(0,0,1.12,1.13,1.14,1.15,1.16+1.25+1.34,1.17,1.18+1.27+1.45)$ & 7 & 3 \\
\hline 91 & $(0,0,1.12,1.13,1.14,1.15,1.16+1.25+1.34,1.17,1.18+1.27+1.36)$ & 7 & 3 \\
\hline 92 & $(0,0,1.12,1.13,1.14,1.15,1.16+1.25+1.34,1.17+1.35,1.18+1.27)$ & 7 & 3 \\
\hline 93 & $(0,0,1.12,1.13,1.14+1.23,1.15+1.24,1.16+1.34,1.17+1.35,1.18)$ & 7 & 3 \\
\hline 94 & $(0,0,1.12,1.13,1.14+1.23,1.15+1.24,1.16+1.34,1.17+1.26,1.18)$ & 7 & 3 \\
\hline 95 & $(0,0,1.12,1.13,1.14+1.23,1.15+1.24,1.16+1.25,1.17,1.18+1.45)$ & 7 & 3 \\
\hline 96 & $(0,0,1.12,1.13,1.14+1.23,1.15+1.24,1.16+1.25,1.17,1.18+1.36)$ & 7 & 3 \\
\hline 97 & $(0,0,1.12,1.13,1.14+1.23,1.15+1.24,1.16+1.25,1.17+1.35,1.18)$ & 7 & 3 \\
\hline 98 & $(0,0,1.12,1.13,1.14+1.23,1.15+1.24,1.16+1.25+1.34,1.17,1.18)$ & 7 & 3 \\
\hline
\end{tabular}


in the first row of Table 3 is meant to encode the metric Lie algebra $(n,\langle\cdot, \cdot\rangle)$ with orthonormal basis $B=\left\{X_{i}\right\}_{i=1}^{8}$ and bracket relations

$$
\begin{array}{cc}
{\left[X_{2}, X_{3}\right]=\sqrt{45} X_{5},} & {\left[X_{2}, X_{4}\right]=\sqrt{14} X_{6}} \\
{\left[X_{2}, X_{5}\right]=\sqrt{91} X_{7},} & {\left[X_{3}, X_{4}\right]=\sqrt{91} X_{7}} \\
{\left[X_{1}, X_{7}\right]=\sqrt{136} X_{8},} & {\left[X_{2}, X_{6}\right]=\sqrt{29} X_{8}} \\
{\left[X_{3}, X_{5}\right]=\sqrt{14} X_{8},} & {\left[X_{1}, X_{8}\right]=\sqrt{104} X_{9}} \\
{\left[X_{2}, X_{7}\right]=4 X_{9} .}
\end{array}
$$

4.2.1. Candidates of Nilsoliton Metrics. Table 5 illustrates how many possible candidates of Lie algebras appear in dimension 9 up to the nullity of its Gram matrix. The algebras illustrated in Table 4 are possible candidates of nilsoliton metric Lie algebras with ordered type of derivations in dimension 8 . Here, as an example we give potential Lie algebra structures when the nullity of their corresponding Gram matrices are 3, 6 and 8 in Tables 6, 7, and 8 respectively for dimension nine.

\section{Conclusion}

In this work, we have focused on nilpotent metric Lie algebras of dimensions eight and nine with ordered type of derivations. We have given specific Jacobi identity conditions for Lie algebras which allowed us to simplify the Jacobi identity condition. We have classified nilsoliton metric Lie algebras for the corresponding Gram matrix $U$ being invertible and noninvertible. For dimension 8, we have focused on nilsoliton metric Lie algebras with noninvertable Gram matrix which leads to more than one solution for $U v=[1]$. We have proved that if the nilpotent Lie algebra admits a soliton metric with corresponding Gram matrix being noninvertible, all the solutions of $U v=$ [1] correspond to a unique derivation. This theorem has allowed us to omit several cases that come from nonordered eigenvalues without considering Jacobi condition. Moreover, we have classified some nilsoliton metric Lie algebras with derivation types $1<2<\cdots<n$ and provided some candidates that may be classified. We are currently working on an algorithm that provides a full list of classifications for dimensions eight and nine.

\section{Appendix}

See Tables 5, 6, 7, and 8 .

\section{Conflict of Interest}

Author declares that she has no competing interest.

\section{Acknowledgment}

Hülya Kadıoğlu would like to thank the Idaho State University Department of Mathematics, in particular department chair Professor Dr. Robert Fisher Jr. and Professor Dr. Tracy
L. Payne for their advices and technical supports during her research.

\section{References}

[1] W. A. de Graaf, "Classification of 6-dimensional nilpotent Lie algebras over fields of characteristic not 2," Journal of Algebra, vol. 309, no. 2, pp. 640-653, 2007.

[2] J. Lauret, "Finding Einstein solvmanifolds by a variational method," Mathematische Zeitschrift, vol. 241, no. 1, pp. 83-99, 2002.

[3] C. Will, "Rank-one Einstein solvmanifolds of dimension 7," Differential Geometry and its Applications, vol. 19, no. 3, pp. 307318, 2003.

[4] H. Kadioglu and T. L. Payne, "Computational methods for nilsoliton metric Lie algebras I," Journal of Symbolic Computation, vol. 50, pp. 350-373, 2013.

[5] E. A. Fernández-Culma, "Classification of 7-dimensional Einstein nilradicals," Transformation Groups, vol. 17, no. 3, pp. 639656, 2012.

[6] E. A. Fernández-Culma, "Classification of 7-dimensional Einstein nilradicals II," Transformation Groups. In press.

[7] J. Lauret and C. Will, "Einstein solvmanifolds: existence and non-existence questions," Mathematische Annalen, vol. 350, no. 1, pp. 199-225, 2011.

[8] Y. Nikolayevsky, "Einstein solvmanifolds with a simple Einstein derivation," Geometriae Dedicata, vol. 135, pp. 87-102, 2008.

[9] R. M. Arroyo, "Filiform nilsolitons of dimension 8," The Rocky Mountain Journal of Mathematics, vol. 41, no. 4, pp. 1025-1043, 2011.

[10] J. Lauret, "Einstein solvmanifolds and nilsolitons," in New Developments in Lie Theory and Geometry, vol. 491 of Contemporary Mathematics, pp. 1-35, American Mathematical Society, Providence, RI, USA, 2009.

[11] T. L. Payne, "The existence of soliton metrics for nilpotent Lie groups," Geometriae Dedicata, vol. 145, pp. 71-88, 2010. 


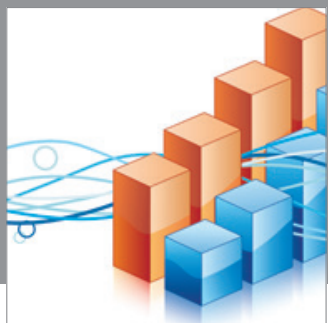

Advances in

Operations Research

mansans

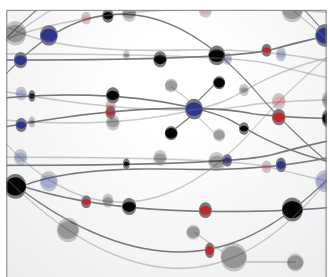

The Scientific World Journal
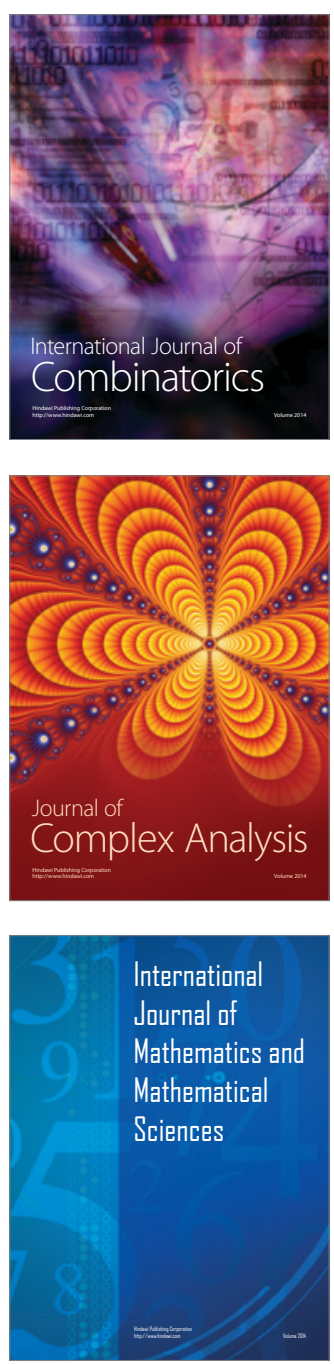
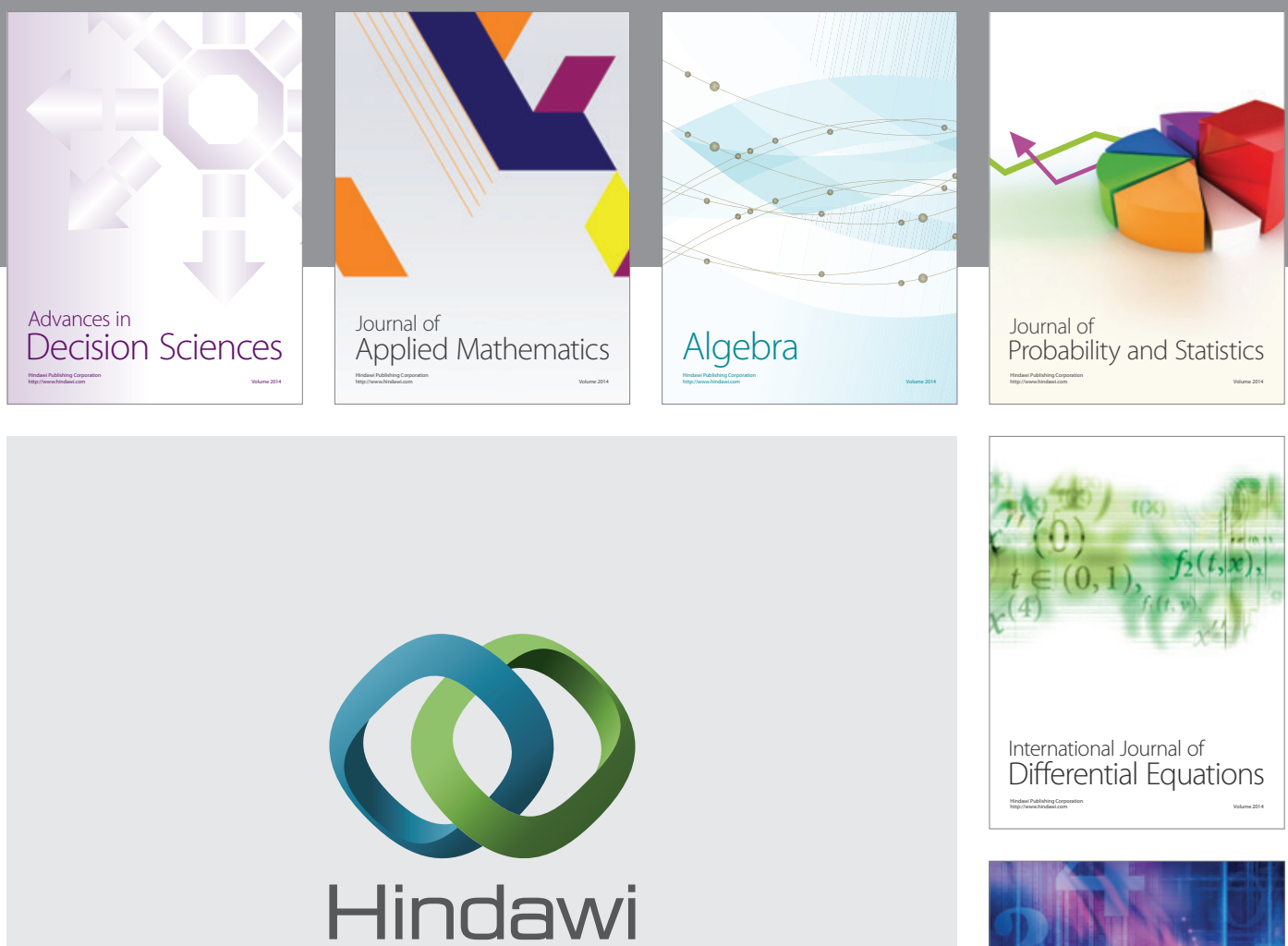

Submit your manuscripts at http://www.hindawi.com
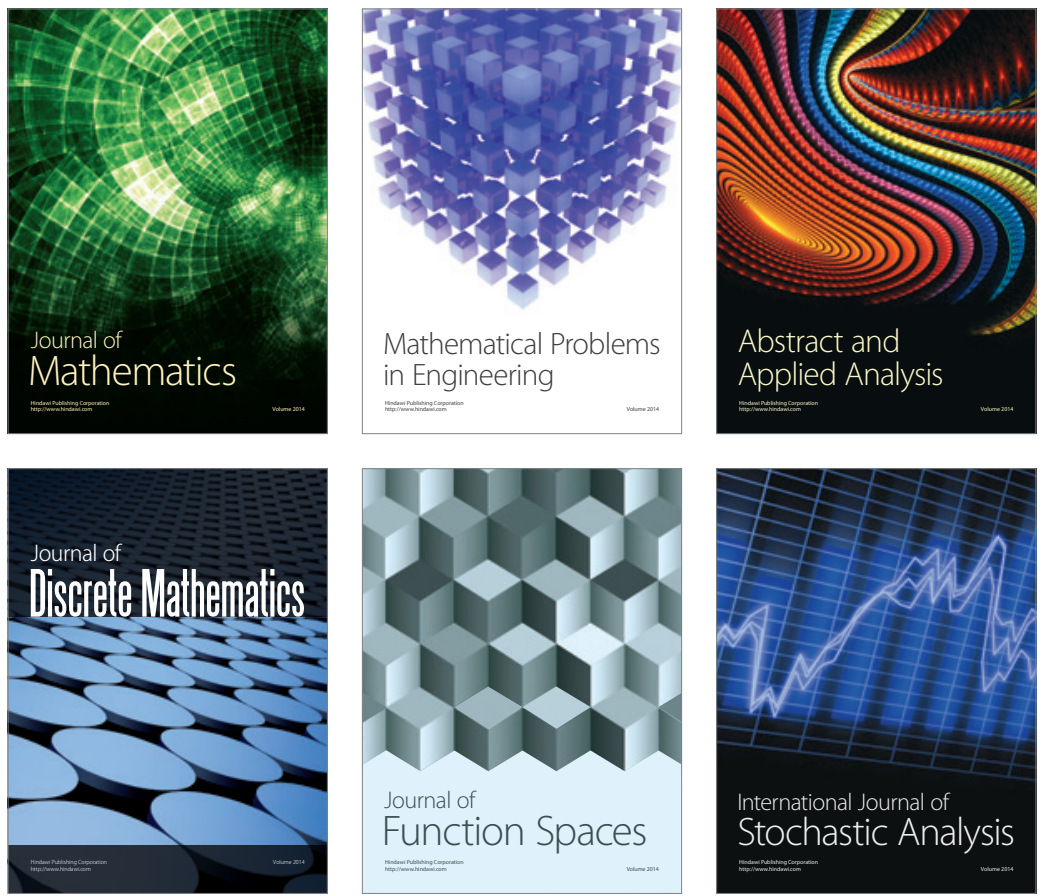

Journal of

Function Spaces

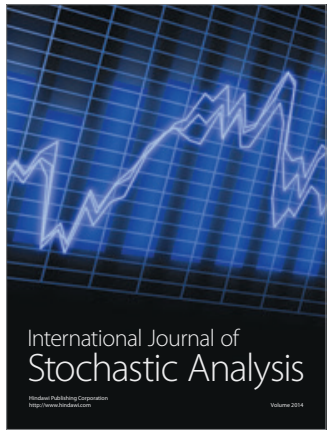

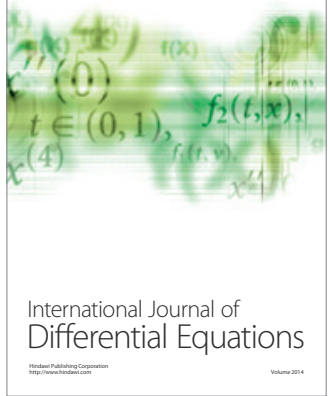
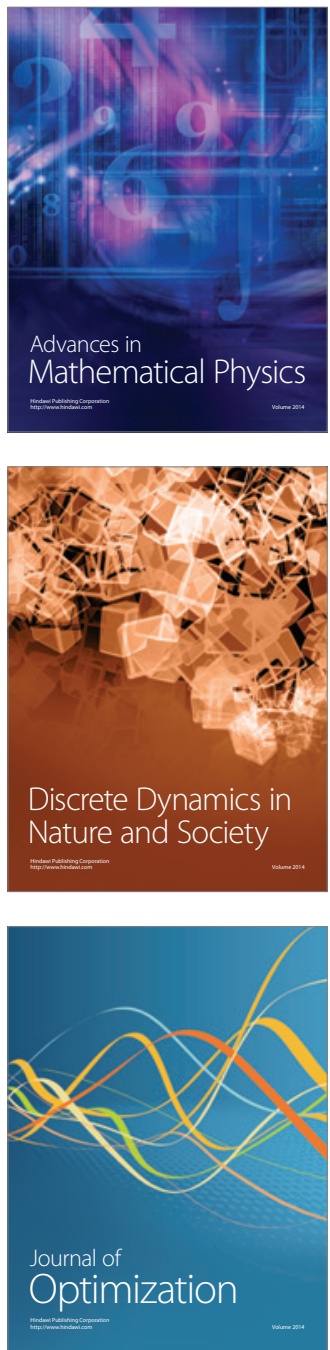